\title{
Preservação da Fertilidade em Mulheres Jovens com Carcinoma do Endométrio: A Propósito de um Caso Clínico
}

\section{Endometrial Cancer and Fertility Preservation in Young Women: A Case Report}

José Silva Pereira ${ }^{1}$, Teresa Fragaํㅣ Julieta Silva², João Paulo Fernandes ${ }^{3}$, Cláudia Monteiro ${ }^{3}$, João $\mathrm{Cruz}^{4}$, Mónica Ferreira ${ }^{4}$, Paula Borralho ${ }^{4}$, Álvaro Dias ${ }^{5}$, Catarina Vasconcelos ${ }^{5}$

\section{RESUMO}

Nos últimos anos, um número crescente de mulheres adia o seu projeto reprodutivo para idades mais avançadas, sendo por isso cada vez mais importante que se avalie a possibilidade e a segurança de tratamentos conservadores que preservem a fertilidade em pacientes jovens com carcinoma do endométrio. O tratamento recomendado para o carcinoma do endométrio é a histerectomia total com salpingo-ooforectomia, o que interfere, como é óbvio, na fertilidade da mulher, não sendo por isso recomendável em mulheres que pretendem preservar a sua função reprodutora. Em alternativa, o tratamento conservador pode ser uma opção de exceção em casos bem selecionados.

Contudo, a decisão de efetuar tratamento conservador não está isenta de riscos, nomeadamente os riscos de estadiar e/ou tratar inadequadamente o carcinoma do endométrio e os riscos de não diagnosticar tumores ováricos síncronos ou metastáticos. Além disso, não há consenso quanto ao tratamento ideal por quanto tempo deve ser prolongado, como efetuar a vigilância destas doentes e qual o resultado oncológico a longo prazo.

O caso presente, no conhecimento dos autores, é o primeiro documentado em Portugal de gravidez após tratamento conservador de carcinoma do endométrio, numa mulher jovem.

PALAVRAS-CHAVE: Neoplasias do Endométrio; Preservação da Fertilidade

\section{ABSTRACT}

As more women choose to defer childbearing until later in life, the feasibility and safety of fertility-sparing endometrial cancer management, have been increasingly studied. The standard treatment for endometrial cancer involves hysterectomy and bilateral salpingo-oophorectomy, which precludes future fertility and is undesirable for women who wish to maintain their reproductive potential. However, the decision of conservative management is associated with some risks namely the oncolo- 
gic risks of inadequately staged and treated endometrial cancer and the risk of missing a synchronous or metastatic ovarian cancer, and the lack of uniformity in the medical management and follow-up.

As far as we know this is the first well succeeded case in Portugal, for conservative management of endometrial carcinoma with subsequent pregnancy in a young woman.

KEYWORDS: Endometrial Neoplasms; Fertility Preservation

\section{INTRODUÇÃO}

O carcinoma do endométrio (CE) é a neoplasia genital feminina mais frequente nos países desenvolvidos, e afeta sobretudo mulheres na pós-menopausa. Contudo, cerca de 14\% das mulheres encontram-se na pré-menopausa e 4\% serão diagnosticadas antes dos 40 anos de idade.

À medida que as mulheres protelam o seu projeto reprodutivo, maior é a probabilidade de se confrontarem com este tipo de problema. Estima-se que atualmente cerca de uma em cada 12 mulheres terão a sua primeira gravidez aos 35 anos, comparado com uma em cada 100 no ano de 1970.

A maior parte dos casos diagnosticados nestas mulheres mais jovens, são, contudo, neoplasias bem diferenciadas e em estádios precoces com um prognóstico excelente e estão frequentemente associadas a obesidade ou síndroma do ovário poliquístico (hiperestimulação estrogénica). ${ }^{1}$ Num estudo retrospetivo do Geneva Cancer Registry apenas 18\% (oito de 44) das mulheres de menos de 45 anos reuniam critérios simultâneos de carcinoma endometrial em estádio I e bem diferenciados (G1), sendo por isso candidatas possíveis a tratamento conservador. $^{2}$

O tratamento recomendado para o CE é a histerectomia com anexectomia bilateral, e a decisão de se efetuar um tratamento conservador nestas mulheres jovens levanta alguns problemas, nomeadamente os riscos inerentes a um estadiamento e tratamento inadequados e a associação com tumores síncronos ou metastáticos dos ovários.

As doentes devem ser criteriosamente selecionadas, utilizando critérios muito restritos (CE grau 1, sem invasão linfovascular, estádio inicial sem invasão do miométrio, altamente motivadas para manter o seu potencial reprodutivo) e devem ser devidamente informadas que se trata de um tratamento não estabelecido para o CE, e quais os riscos a ele inerentes.

Apesar de vários grupos descreverem a segurança da terapêutica hormonal para tratamento do CE em estágio inicial, ${ }^{3-7}$ não existe um consenso atual sobre qual o tratamento ideal, a sua dosagem, a duração do mesmo, e quais as modalidades de vigilância e da sua frequência após o tratamento.
Os dois regimes mais utilizados são o acetato de medroxiprogesterona 500-600 mg por dia e o acetato de megestrol 160 mg diários. A eficácia destes regimes varia de $26 \%$ a $89 \%$ nos casos de tumores que expressam recetores de estrogénio e progesterona. 8-14

Como alternativa, alguns estudos reportam que a utilização do dispositivo intrauterino com levonorgestrel (SIU) pode obter respostas entre 40\% a 100\% nas muIheres pré-menopáusicas com estádios precoces de CE bem diferenciado, ${ }^{9-12}$ havendo uma meta-análise que demonstrou menores efeitos secundários do SIU (menor aumento de peso, menos alterações do sono, do humor e da libido, menos enxaquecas), quando comparado com os progestativos orais. ${ }^{11}$

As recidivas são frequentes e podem atingir os 50\%. As doentes submetidas a tratamento conservador devem ser vigiadas com histeroscopia e biópsia do endométrio de três em três meses. ${ }^{12}$

O sucesso obstétrico nestas estratégias alcança 57,7\% de gravidez alcançada nesta população, com 86,6\% de nados vivos.

Após terem cumprido o seu desejo reprodutivo, devem ser submetidas a tratamento cirúrgico definitivo.

\section{CASO CLÍNICO}

Mulher com 36 anos de idade, com uma interrupção voluntária de gravidez aos 27 anos, asmática, com obesidade tipo 1 (IMC = 34,8), a quem em consulta de rotina pré-concecional foi detetado um espessamento endometrial. Foi submetida a histeroscopia com biópsia que revelou pólipo fibroglandular do endométrio. Efetuou então uma ressetoscopia, cujo diagnóstico histológico revelou um adenocarcinoma endometrioide G1 (Fig. 1).

A doente foi proposta para histerectomia total com anexectomia bilateral e foi encaminhada para uma Unidade de Infertilidade para colheita e preservação de oócitos (na perspetiva de utilização futura de mãe de substituição).

Entretanto, a doente veio à nossa consulta de Ginecologia Oncológica procurando uma segunda opinião, e foi então informada de que lhe tinha sido proposto o trata- 


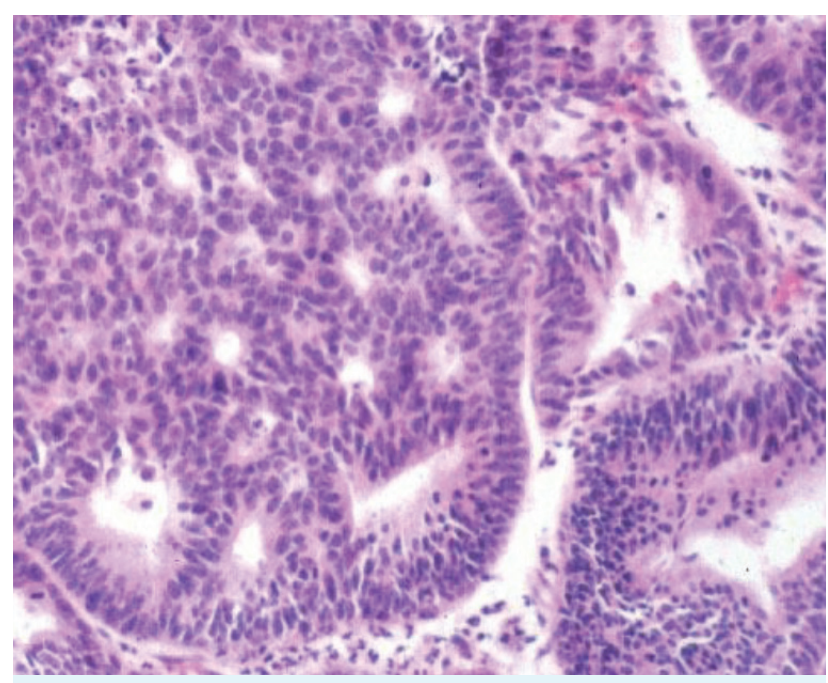

FIGURA 1. Adenocarcinoma endometrioide bem diferenciado (G1).
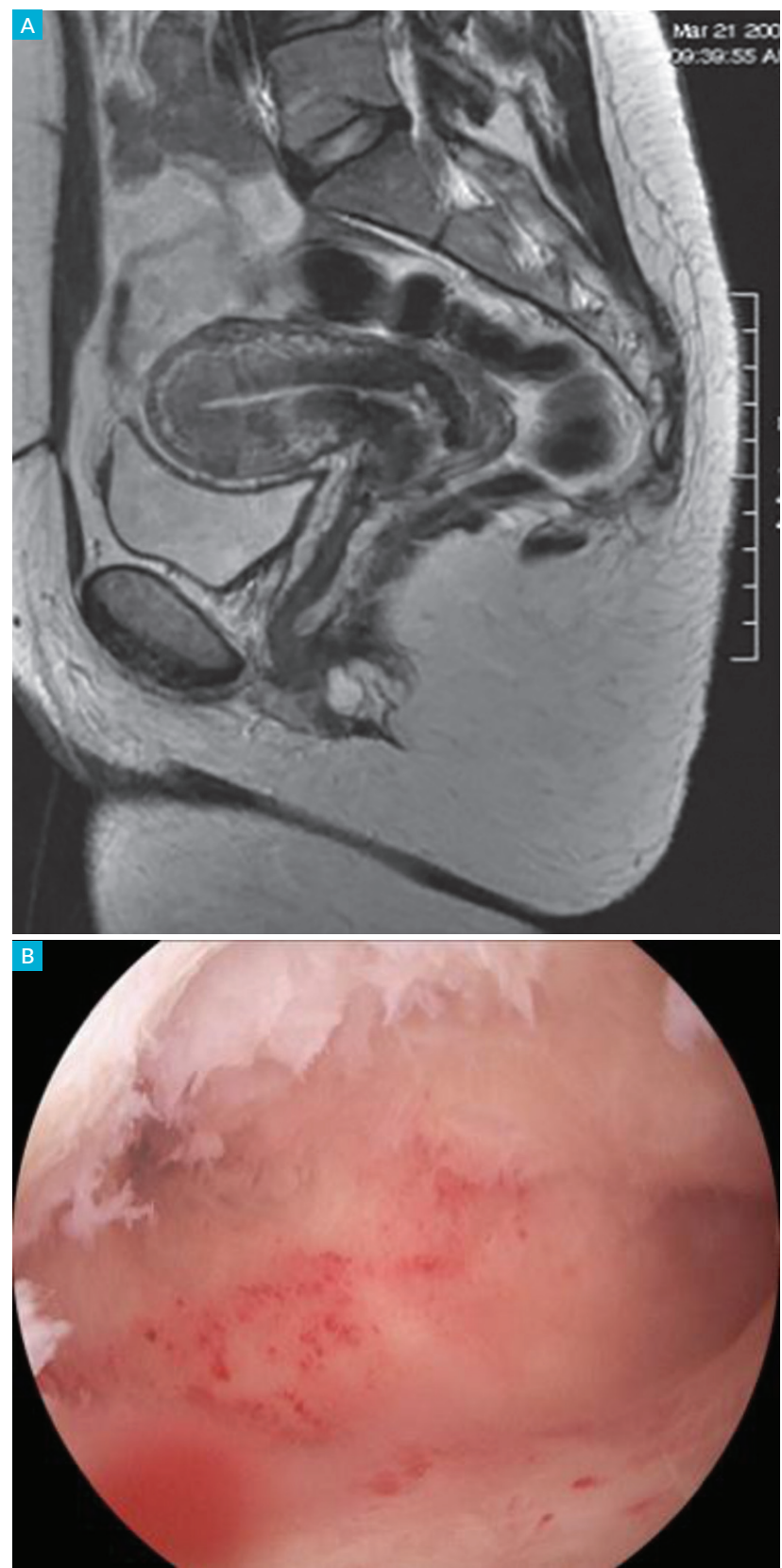

FIGURA 2. A. RM sem sinais de doença intra ou extrauterina. B. Histeroscopia com cavidade livre e endométrio secretor. mento correto para a sua situação, mas foi também informada da hipótese de tratamento conservador e dos riscos inerentes a essa atitude.

Solicitámos que a doente ponderasse e avaliasse a sua situação durante uma semana, e a doente decidiu optar por tratamento conservador, consciente dos riscos de estar a fazer um tratamento inadequado e da possibilidade de progressão da doença.

Foi realizada uma ressonância magnética (RM) abdominal e pélvica, que revelou a presença de adenomiose, sem sinais de doença endometrial ou miometrial ou doença extrauterina (Fig. 2A). Efetuou ainda uma histeroscopia com biópsia que revelou uma cavidade uterina livre (Fig. 2B), e a biópsia revelou uma histologia de endométrio secretor inicial.

Considerada assim como uma boa candidata para tratamento conservador, foi-lhe inserido um dispositivo intrauterino com levonorgestrel (Mirena ${ }^{\circledR}$, Bayer).

O plano de vigilância estipulado com a doente foi de avaliação histeroscópica com biópsia, de três em três meses.

Após três resultados negativos, foi retirado o Mirena ${ }^{\circledR} \mathrm{e}$ a doente foi informada da possibilidade de tentar engravidar. A gravidez aconteceu três meses após remoção do dispositivo (Fig. 3).

O próximo passo será efetuar tratamento cirúrgico definitivo após o parto.

\section{DISCUSSÃO}

O tratamento conservador do carcinoma do endométrio G1 em estádio clínico I em mulheres jovens que desejam preservar a fertilidade é geralmente aceite como uma alternativa de curto prazo até ao tratamento cirúrgico definitivo.

O tratamento conservador do CE grau 1, não está isento de riscos oncológicos, e, ainda que raramente, possa estar associado a envolvimento ganglionar pélvico (3\%) ou lombo-aórtico (1,7\%), invasão profunda do miométrio (9\%), metástases ováricas (6\%) ou neoplasias síncronas do ovário (19\%). O risco de progressão da doença durante o tratamento conservador do CE grau 1, pode atingir os 5\% a 6\%. ${ }^{13}$ Em diversos estudos de tratamento com progestativos a eficácia atinge valores de cerca de $90 \% .{ }^{14}$

As doentes devem ser criteriosamente selecionadas com base no grau de diferenciação do tumor G1 e tipo histológico endometrioide, no estádio avaliado em RM sem invasão do miométrio e na ausência de invasão linfovascular, e devem ser devidamente informadas de que o tratamento 


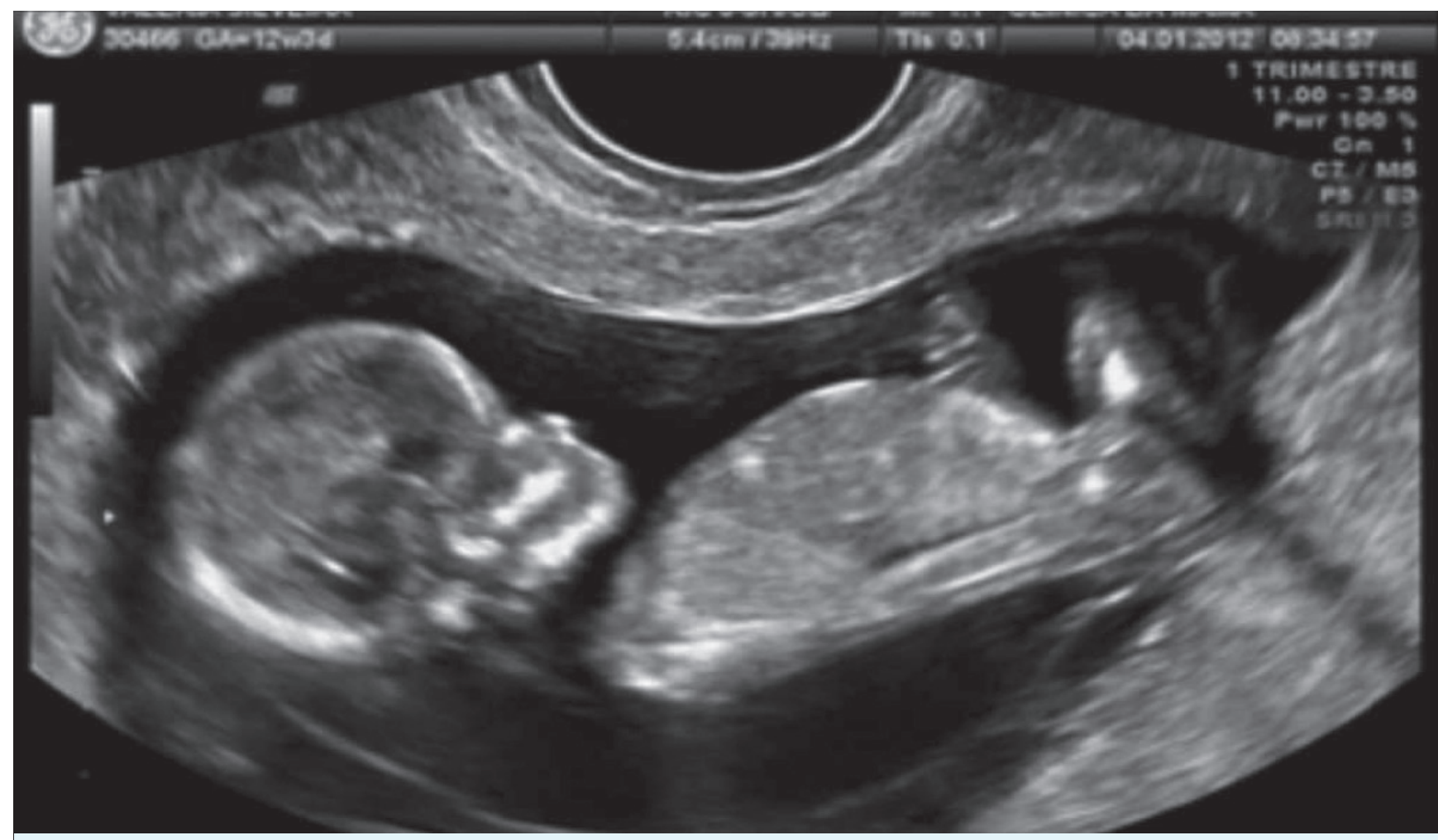

FIGURA 3. Feto com 12 semanas.

adequado nestas situações é a histerectomia total com anexectomia bilateral, devendo estar dispostas a aceitar os riscos inerentes ao tratamento conservador.

Não existe um consenso quanto à melhor modalidade de tratamento, contudo a mais utilizada tem sido a terapêutica com progestativos orais (acetato de megestrol ou acetato de medroxiprogesterona). Alguns estudos recentes, utilizando dispositivos intrauterinos com levonorgestrel, têm demonstrado igual eficácia com menores efeitos adversos. Mazzon et al ${ }^{13}$ utilizaram a associação da ressecção histeroscópica completa do tumor, com progestativos orais, com bons resultados em todas as seis doentes tratadas.

No nosso caso terá sido uma modalidade semelhante, uma vez que a doente tinha sido submetida a uma ressetoscopia e posteriormente colocado um SIU de levonorgestrel.

Mesmo que a regressão do tumor seja completa é importante realçar que o tratamento conservador é uma medida temporária, isto porque a taxa de recorrência é elevada (pode atingir os 50\%), e por isso após a doente conseguir o seu projeto familiar, deverá ser efetuado o tratamento cirúrgico definitivo.

A Ginecologia Oncológica tem um papel importante na avaliação destas doentes e na orientação para tratamentos direcionados para preservação da fertilidade (vitrificação de ovócitos ou criopreservação de embriões ou congelação de tecido ovárico). Nos casos de insucesso em que é necessária a histerectomia, a hipótese de realizar o desejo dessas mulheres passa por técnicas mais sofisticadas e menos disponíveis (transplante uterino) ou mães de substituição (já permitida pela legislação portuguesa).

CONFLITOS DE INTERESSE: Os autores declaram não ter qualquer conflito de interesse na realização do presente trabalho.

FONTES DE FINANCIAMENTO: Não houve qualquer fonte de financiamento na realização do presente trabalho.

CONFIDENCIALIDADE DOS DADOS: Os autores d claram ter seguido os protocolos da sua instituição acerca da publicação dos dados de doentes.

PROTEÇÃO DE PESSOAS E ANIMAIS: Os autores declaram que os procedimentos seguidos na elaboração do presente trabalho estão em conformidade com as normas das comissões de investigação clínica e de ética, bem como da declaração de Helsínquia e da Associação Médica Mundial.

CONFLICTS OF INTEREST: The authors declare that they have no conflicts of interest.

FINANCIAL SUPPORT: This work has not received any contribution, grant or scholarship.

CONFIDENTIALITY OF DATA: The authors declare that they have followed the protocols of their work center on the publication of data from patients. 
PROTECTION OF HUMAN AND ANIMAL SUBJECTS: The authors declare that the procedures followed were in accordance with the regulations of the relevant clinical research ethics committee and with those of the Code of Ethics of the World Medical Association (Declaration of Helsinki).

\section{REFERÊNCIAS}

1. Harris H, Terry K. Polycystic ovary syndrome and risk of endometrial, ovarian, and breast cancer: a systematic review. Fertil Res Pract. 2016;2:14

2. Navarria I, Usel M, Rapiti E, Neyroud-Caspar I, Pelte MF, Bouchardy C, et al. Young patients with endometrial cancer: How many could be eligible for fertility-sparing treatment? Gynecol Oncol. 2009;114:448-51.

3. Bakkum-Gamez J, Kalogera E, Keeney G, Mariani A, Podratz K, Dowdy S. Conservative management of atypical hyperplasia and grade I endometrial carcinoma: Review of the literature and presentation of a series. J Gynecol Surg. 2012;28:262-9.

4. Gotlieb WH, Beiner ME, Shalmon B, Korach Y, Segal Y, Zmira $\mathrm{N}$, et al. Outcome of fertility-sparing treatment with progestins in young patients with endometrial cancer. Obstet Gynecol. 2003;102:718-25.

5. Jobo T, Imai M, Kawaguchi M, Kenmochi M, Kuramoto $\mathrm{H}$. Successful conservative treatment of endometrial carcinoma permitting subsequent pregnancy: report of two cases. Eur J Gynaecol Oncol. 2000;21:119-22.

6. Ramirez PT, Frumovitz M, Bodurka DC, Sun CC, Levenback C. Hormonal therapy for the management of grade 1 endometrial adenocarcinoma: a literature review. Gynecol Oncol. 2004; $95: 133-8$.
7. Park JY, Kim DY, Kim JH, Kim YM, Kim KR, Kim YT, et al. Long-term oncologic outcomes after fertility-sparing management using oral progestin for young women with endometrial cancer (KGOG. 2002). Eur J Cancer. 2013;49:868-74.

8. Kudesia R, Singer T, Caputo TA, Holcomb KM, Kligman I, Rosenwaks Z, et al. Reproductive and oncologic outcomes after progestin therapy for endometrial complex atypical hyperplasia or carcinoma. Am J Obstet Gynecol. 2014;210:255.e1-4.

9. Mentrikoski MJ, Shah AA, Hanley KZ, Atkins KA. Assessing endometrial hyperplasia and carcinoma treated with progestin therapy. Am J Clin Pathol. 2012;138:524-34.

10. Wheeler DT, Bristow RE, Kurman RJ. Histologic alterations in endometrial hyperplasia and well-differentiated carcinoma treated with progestins. Am J Surg Pathol. 2007;31:988-98.

11. Gallos ID, Shehmar M, Thangaratinam S, Papapostolou TK, Coomarasamy A. Gupta JK. Oral progestogens vs levonorgestrel-releasing intrauterine system for endometrial hyperplasia: a systematic review and metaanalysis. Am J Obstet Gynecol. 2010;203:547.e1-10

12. Gunderson CC, Fader AN, Carson KA, Bristow RE. Oncologic and reproductive outcomes with progestin therapy in women with endometrial hyperplasia and grade 1 adenocarcinoma: a systematic review. Gynecol Oncol. 2012;125:477-82.

13. Mazzon I, Corrado G, Masciullo V, Morricone D, Ferrandina G, Scambia G. Conservative surgical management of stage IA endometrial carcinoma for fertility preservation. Fertil Steril. 2010;93:1286-9.

14. Carneiro M, Lamaita R, Ferreira M, Silva-Filho A. Fertility preservation in endometrial cancer: is it safe? Review of the literature. JBRA Assist Reprod. 2016;20:232-9. 